Supplementary Information:

\title{
Quantum Emitter Localisation in Layer-Engineered Hexagonal Boron Nitride
}

James Callum Stewart ${ }^{1}$, Ye Fan ${ }^{1}$, John S. H. Danial ${ }^{2}$, Alexander Goetz ${ }^{3}$, Adarsh S. Prasad ${ }^{3}$, Oliver J. Burton $^{1}$, Jack A. Alexander-Webber ${ }^{1}$, Steven F. Lee ${ }^{2}$, Sarah M. Skoff ${ }^{3}$, Vitaliy Babenko ${ }^{1}$, and Stephan Hofmann ${ }^{1, *}$

${ }^{1}$ Department of Engineering, University of Cambridge, $9 \mathrm{JJ}$ Thomson Avenue, Cambridge, CB3 OFA, United Kingdom

${ }^{2}$ The Yusuf Hamied Department of Chemistry, University of Cambridge, Lensfield Road, Cambridge, CB2 1EW, United Kingdom

${ }^{3}$ Institute of Atomic and Subatomic Physics, Vienna University of Technology, Stadionallee 2, 1020 Vienna, Austria

*E-mail:sh315@cam.ac.uk 
a

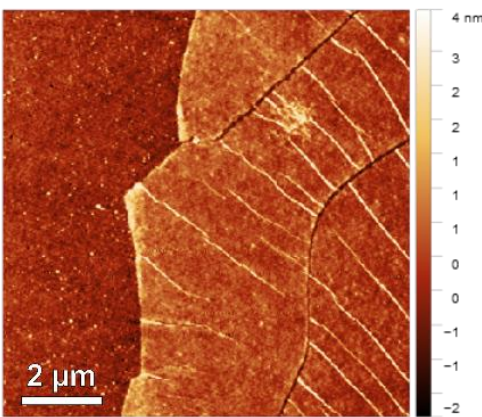

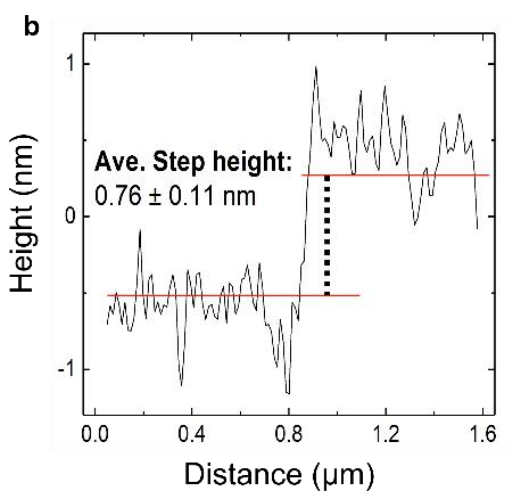

C

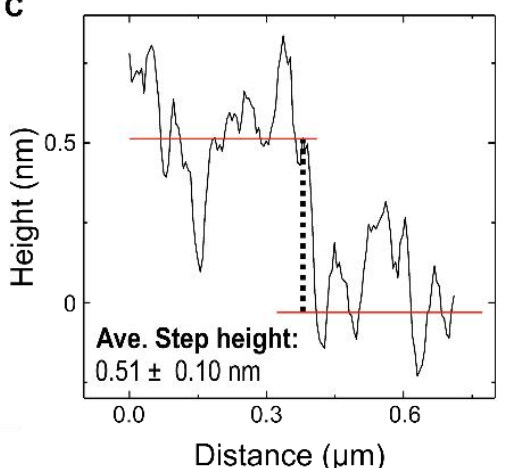

Figure S1: Step-height of ML hBN: (a) Representative AFM image of the edge of as-transferred monolayer $h B N$. Height profile plots of (b) as-transferred $h B N$ and (c) $O-h B N$, with extrapolated average values and their standard deviation.

The AFM data in Figure S1 indicates the presence of transfer residue on the surface of as-transferred ML hBN. The majority of the PVA used for transfer is dissolved during the water treatment of the sample (see Methods). However, as shown in Figure 3a, a detectable signature, attributable to the C$\mathrm{H}$ vibrational mode of the polymer residue, is still present. ${ }^{1,2}$ While the residue coverage of the $\mathrm{hBN}$ is fairly uniform, its thickness can vary from sample to sample and across each individual sample; shown by the Raman map (see Figure S3a). The mean value for the increased step-height of as-transferred ML hBN was determined by sampling numerous flake edges over 2 samples and taking the mean. The value of $0.76 \pm 0.11 \mathrm{~nm}$ (where the error was taken as the standard deviation of the dataset)is distinct to the value of the O-hBN sample shown in Fig. S1c, where the removal of residue and conforming of the film to the $\mathrm{SiO}_{2}$ substrate from the air anneal decreases the step height to $0.51 \pm 0.10 \mathrm{~nm}$. 

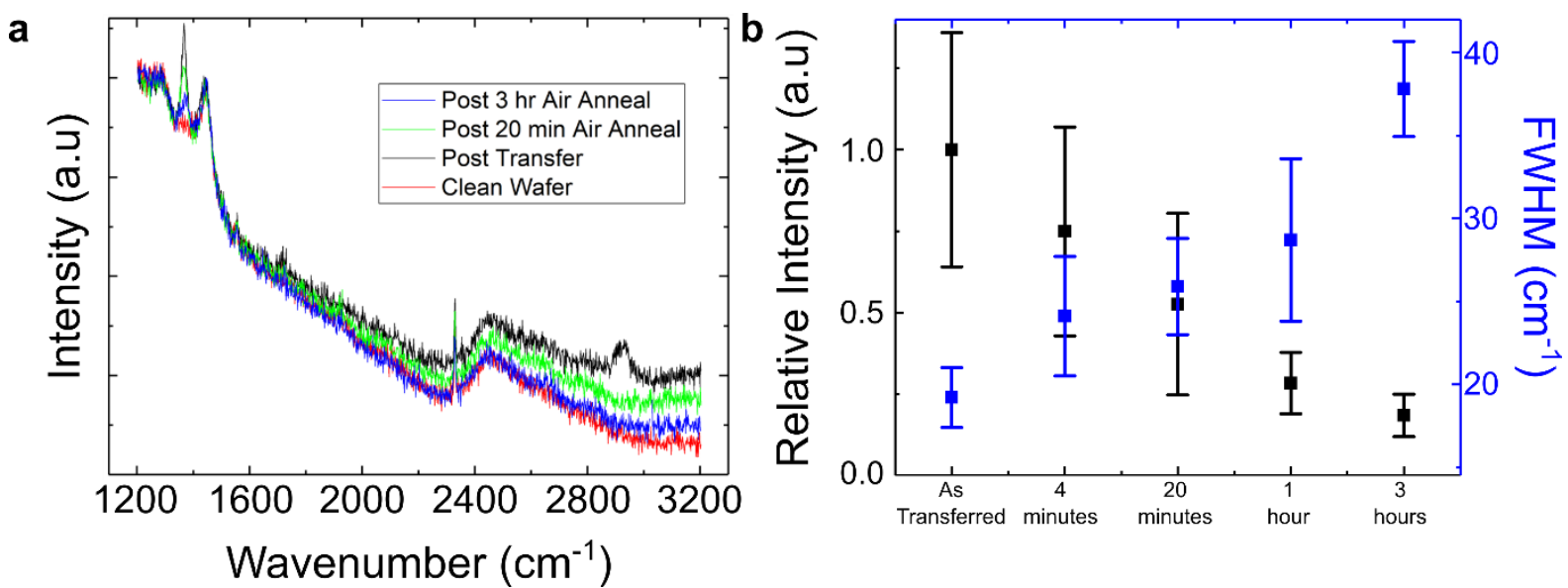

Figure S2: Polymer Removal: (a) Representative Raman spectra of as-transferred ML hBN (black) compared with a clean $\mathrm{Si}_{/} \mathrm{SiO}_{2}$ wafer (red curve), highlighting how the background evolves with two different air anneal times: 20 minutes (green) used as the standard $O$-step and 3 hours (blue) the time required to closely match the spectra to a clean sample. (b) Plot showing the extent of oxidation after different air annealing times used in this report, as measured by the intensity (black) and FWHM (blue) of the $E_{2 g}$ Raman peak.

Although the residue peak in the Raman spectra, shown in Fig $2 \mathrm{bi}$, is removed after the 20-minute oxygen anneal (green, Fig S2a) there remains some background deviation from the spectra of a clean $\mathrm{Si} / \mathrm{SiO}_{2}$ wafer (red, Fig S2a). The extent of this background varies as the level of residue varies across the hBN ML (Fig S3a) and in some cases a 'clean' (comparable background to clean Si/SiO ${ }_{2}$ ) Raman spectra for ML hBN was achieved after 4 minutes of air annealing (Fig S3b). As discussed in the main text, a compromise was needed to balance cleanliness, variations in residue thickness, and hBN quality/lack of oxidation: 20 minutes was chosen as it was always found to remove the peaks attributable to the residue at $2800-2900 \mathrm{~cm}^{-1}$. And although a background persists, it minimises the damage done to hBN (considering residue thickness), in contrast to the Raman spectra of the $3 \mathrm{hr}$ anneal, which very closely matches the clean $\mathrm{Si} / \mathrm{SiO}_{2}$, but has a significant degradation of the $\mathrm{E}_{2 \mathrm{~g}}$ peak. 
a

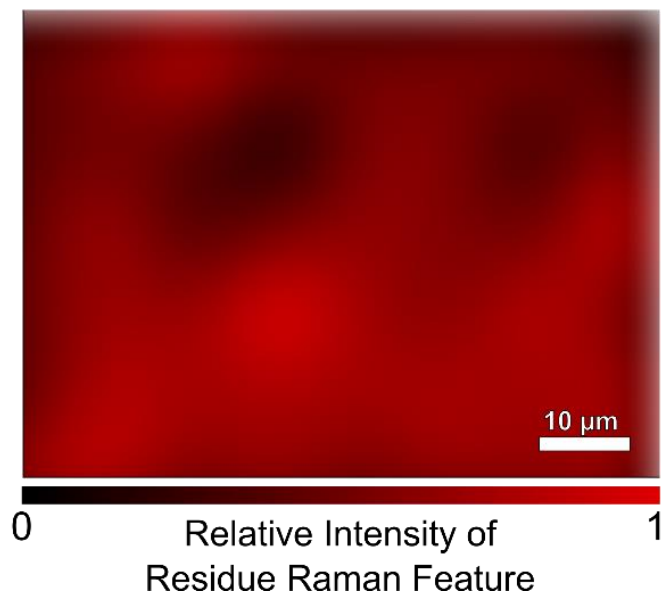

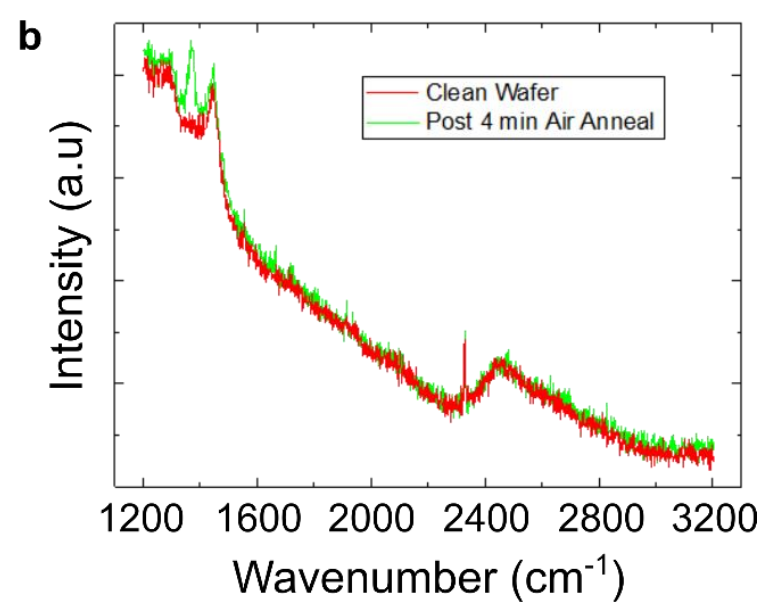

Figure S3: Residue thickness variation: (a) Raman map of the intensity variation (background subtracted) of the feature at $2800-2900 \mathrm{~cm}^{-1}$ attributable to $\mathrm{C}-\mathrm{H}$ bond vibrations in the polymer residue (see Figure 2bi). (b) Representative Raman spectra highlighting the effect the variation in residue thickness has on the effectiveness of the air anneal. In this example a 4-minute air anneal (green) is sufficiently long to match the cleanliness of the $\mathrm{hBN}$ to a clean $\mathrm{Si}_{/} \mathrm{SiO}_{2}$ wafer (red). However, the $E_{2 g}$ peak degrades significantly more than the average for a 4-minute air anneal. This supports the argument that a carbonaceous coating from the transfer process can act as initial protective layer for $M L h B N$.
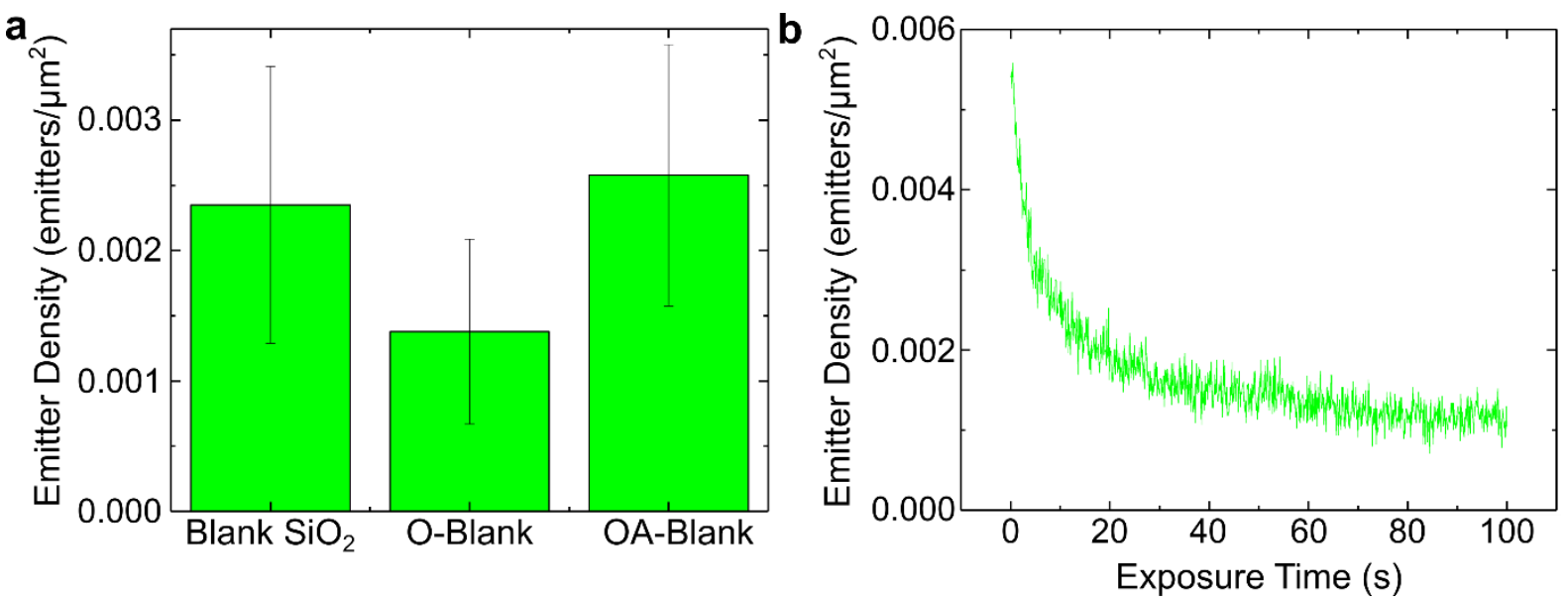

Figure S4: Blank and Residue emission site density baselines: (a) Densities of emission sites detected on blank $\mathrm{Si}_{\mathrm{SiO}}$ wafers, highlighting the inherent environmental contaminating fluorescent species present. Each treatment step was carried out on these blank samples to ensure that potential contaminants from processing were included in the emission density measurements for the relevant $M L ~ h B N$ sample. (c) Bleaching of a dummy transfer sample (see Methods), showing the instability of the emission sites originating from the PVA residue (after water dissolution) and their contribution to the overall density of emission sites on the as-transferred sample. 


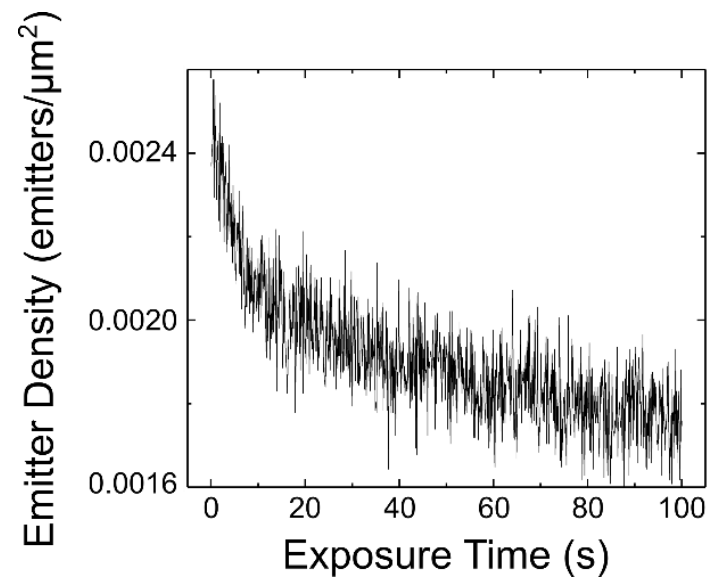

Figure S5: Emitter density of $O-h B N$ under the same laser exposure as the samples in the main text (see Methods). The initial density is comparable to that of a blank $\mathrm{SiO}_{2}$ wafer (Fig. S4), showing that all "emission" sites are due to environmental or substrate contaminants. The maintenance of the lower emitter density shows that the complexes formed in the emitter pre-bleaching of the $\mathrm{O}$-step are stable under illumination.

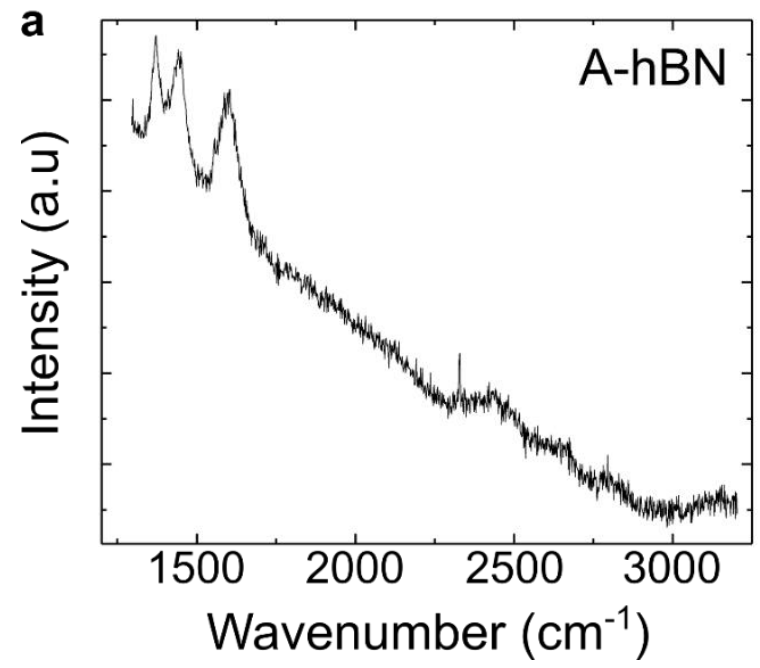

b

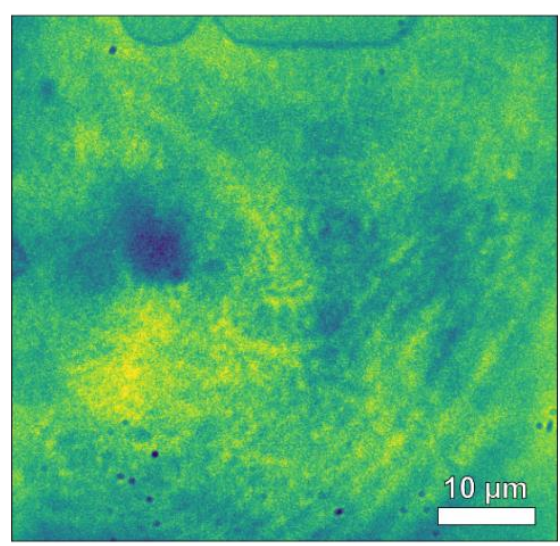

Figure S6: (a) Raman signature of $M L h B N$ sample Ar annealed in the same conditions as those samples in the main text (see Methods), but without a preceding air anneal (A-hBN). The graphitisation of the polymer residue can be clearly seen, with the Gaussian peak fit centred at $1601 \mathrm{~cm}^{-1}$. (b) PL image of the same sample showing bright background, no emitters can be distinguished due to the presence of the graphitised carbon. Features at the top are gold alignment markers. 


\begin{tabular}{|c|c|c|c|c|}
\hline Sample & $\begin{array}{l}\text { Monolayer } 1 \\
\text { (Yellow Boxes show } \\
\text { SPE Host layer) }\end{array}$ & $\begin{array}{l}\text { Monolayer } 2 \text { (Yellow } \\
\text { Boxes show SPE Host } \\
\text { layer) }\end{array}$ & Monolayer 3 & $\begin{array}{l}\text { Structure (blue line hBN } \\
\mathrm{ML} \text {, yellow dot } \\
\text { represents presence of } \\
\text { emitters) }\end{array}$ \\
\hline $\begin{array}{l}\text { O-hBN } \\
\text { (Encapsulation } \\
\text { Layer) }\end{array}$ & $\begin{array}{l}\text { 1. Transferred } \\
\text { to target } \\
\text { 2. Water } \\
\text { dissolution } \\
\text { 3. Air Anneal }\end{array}$ & & & \\
\hline $\begin{array}{l}\text { OA-hBN } \\
\text { (SPE Host } \\
\text { Layer) }\end{array}$ & $\begin{array}{l}\text { 1. Argon } \\
\text { Anneal of } \\
\text { O-HBN ML } \\
\text { without } \\
\text { quantum } \\
\text { emitters } \\
\end{array}$ & & & \\
\hline O/OA-Bi hBN & 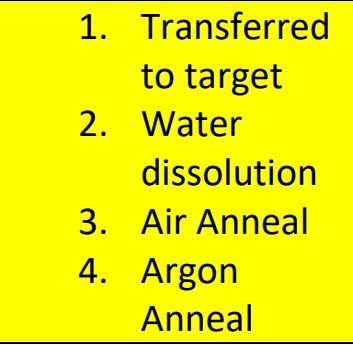 & $\begin{array}{l}\text { 1. Transferred } \\
\text { onto Layer } 1 \\
\text { 2. Water } \\
\text { dissolution } \\
\text { 3. Air Anneal }\end{array}$ & & 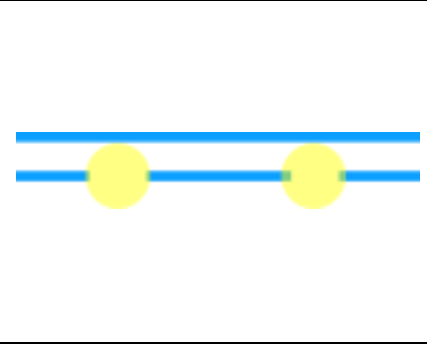 \\
\hline $\begin{array}{l}\text { O/OA/O-Tri } \\
\text { hBN }\end{array}$ & $\begin{array}{l}\text { 1. Transferred } \\
\text { to target } \\
\text { 2. Water } \\
\text { dissolution } \\
\text { 3. Air Anneal }\end{array}$ & 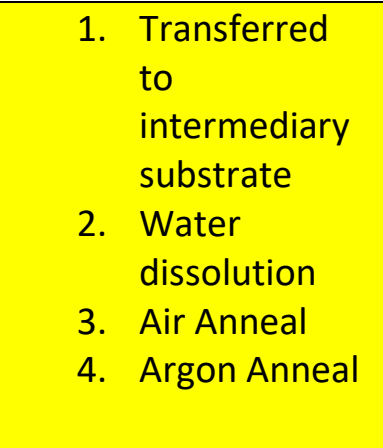 & $\begin{array}{l}\text { 1. Transferred } \\
\text { onto Layer } 2 \\
\text { 2. Use to pick up } \\
\text { Layer } 2 \text { and } \\
\text { transfer both } \\
\text { onto layer } 1 \\
\text { 3. Water } \\
\text { Dissolution } \\
\text { 4. Air Anneal }\end{array}$ & $\square$ \\
\hline
\end{tabular}

Table S1: Overview of assembly and treatments carried out on the different hBN sample structures. 

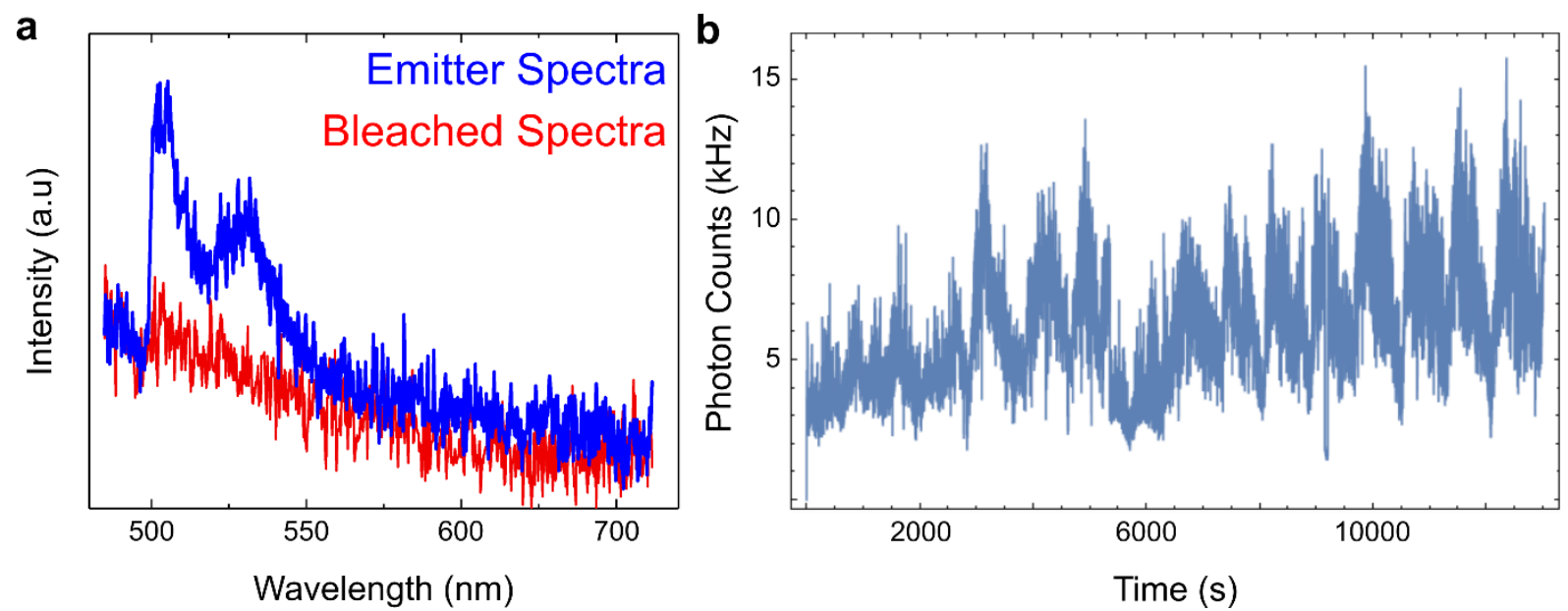

Figure S7: (a) PL spectra showing an example emitter, before and after it has bleached, highlighting that it is the hBN emitters which are bleaching. (b) Raw intensity counts incident on both detectors combined during $g^{2}$ measurements (oscillations are most probably due to polarisation fluctuations in the set-up).
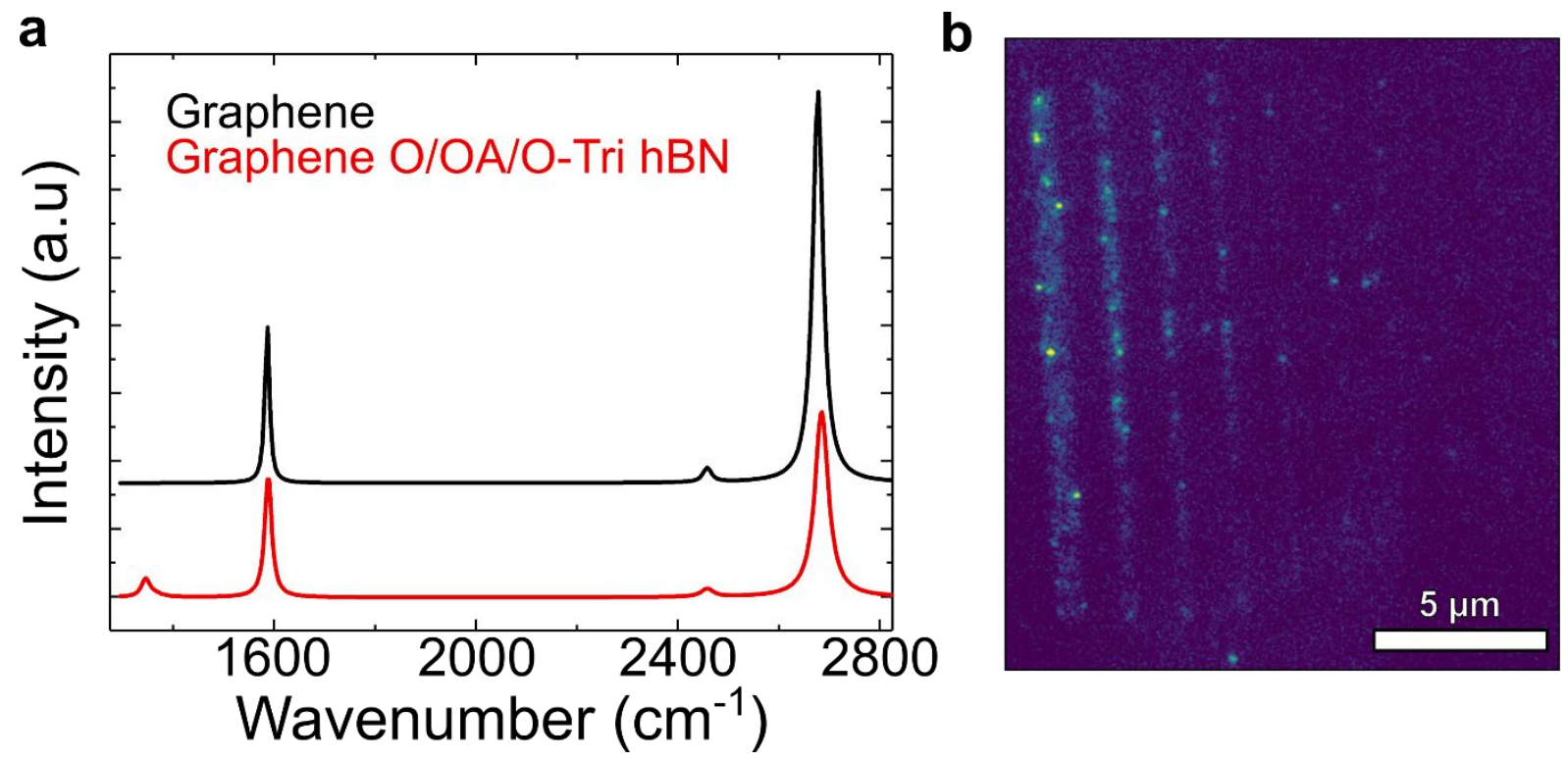

Figure S8: ML hBN Protection: (a) Lorentzian fitted Raman spectra of as-transferred graphene (black) showing the G-peak at $1586 \mathrm{~cm}^{-1}$ and 2D-peak at $2678 \mathrm{~cm}^{-1}$ and graphene after O/OA/O-Tri hBN assembly. The $h B N$ sufficiently protects $M L$ graphene from damage during all treatments, with only a small D-peak developing at $1346 \mathrm{~cm}^{-1}$ visible. The $h B N E_{2 g}$ peak can be seen as a shoulder to the D-peak with a centre at $1370 \mathrm{~cm}^{-1}$. (b) PL image of $M L O A-h B N$ on ML graphene showing that quenching of emission is still maintained after all treatments are carried out, with only a hBN ML serving as graphene protection. 


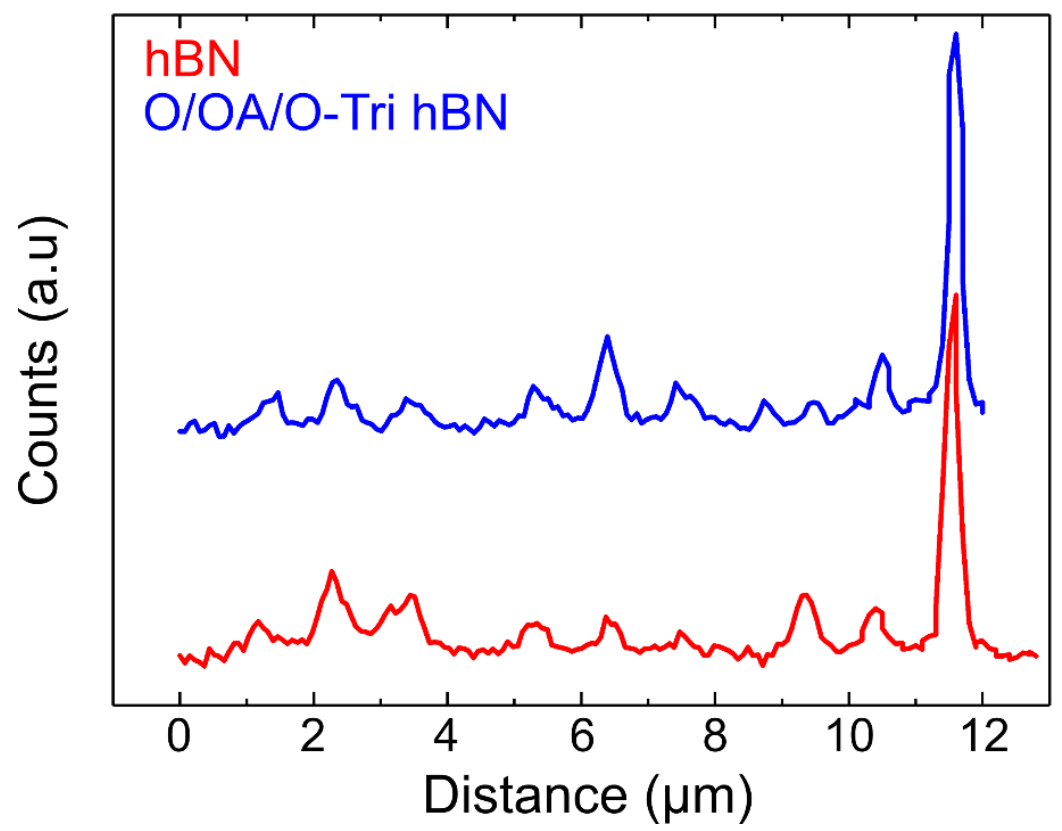

Figure 59: Intensity profile across the first three sets of three lines (as described in main manuscript, Fig. 4) of as transferred $h B N$ and the O/OA/O-Tri hBN layer on the graphene mask. This shows that the noise level on the graphene is lower than within the holes and that there is similarity in both noise levels across the two samples. Also, the intensity of emitters is found to be comparable, with an exemplar emission site showing this in the ninth hole of both samples.
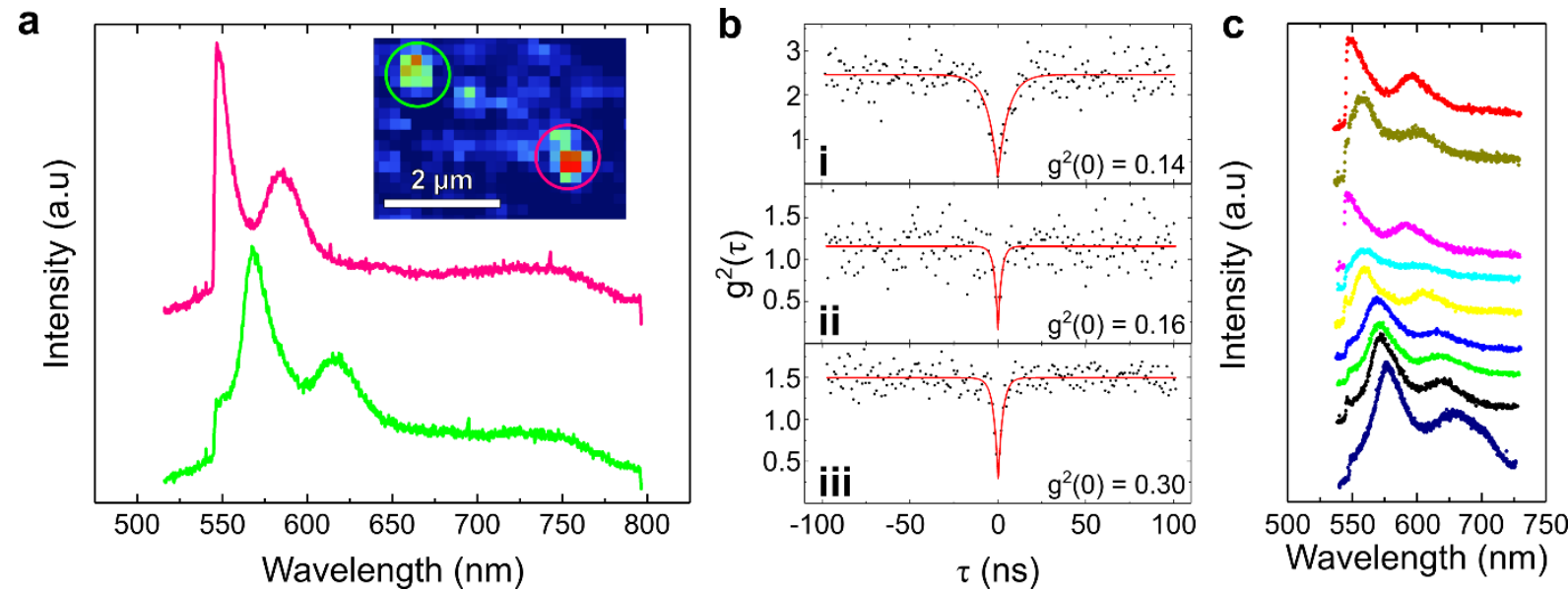

Figure S10: Emitter characterisation of O/OA/O-Tri hBN without graphene mask. (a) Two example spectra showing each of the central wavelengths, $550 \mathrm{~nm}$ (i) and $575 \mathrm{~nm}$ (ii), that the emitters cluster around in these samples. The inset shows the confocal image of each emitter and the cut-off at 550 $\mathrm{nm}$ is due to the long-pass filter mentioned in the main text. (b) Representative second order fluorescence intensity correlation measurements confirming the single-photon nature of the emission sites. Background correction was conducted for each plot and the corresponding values are (i) 0.69 , (ii) 0.90, and (iii) 0.81. A description of the collection parameters and fitting can be found in Fig. 5 and Methods. (c) Further example spectra of emitters from the O/OA/O-Tri hBN sample, both with and without graphene, used to make the histogram in Fig. $5 c$. 


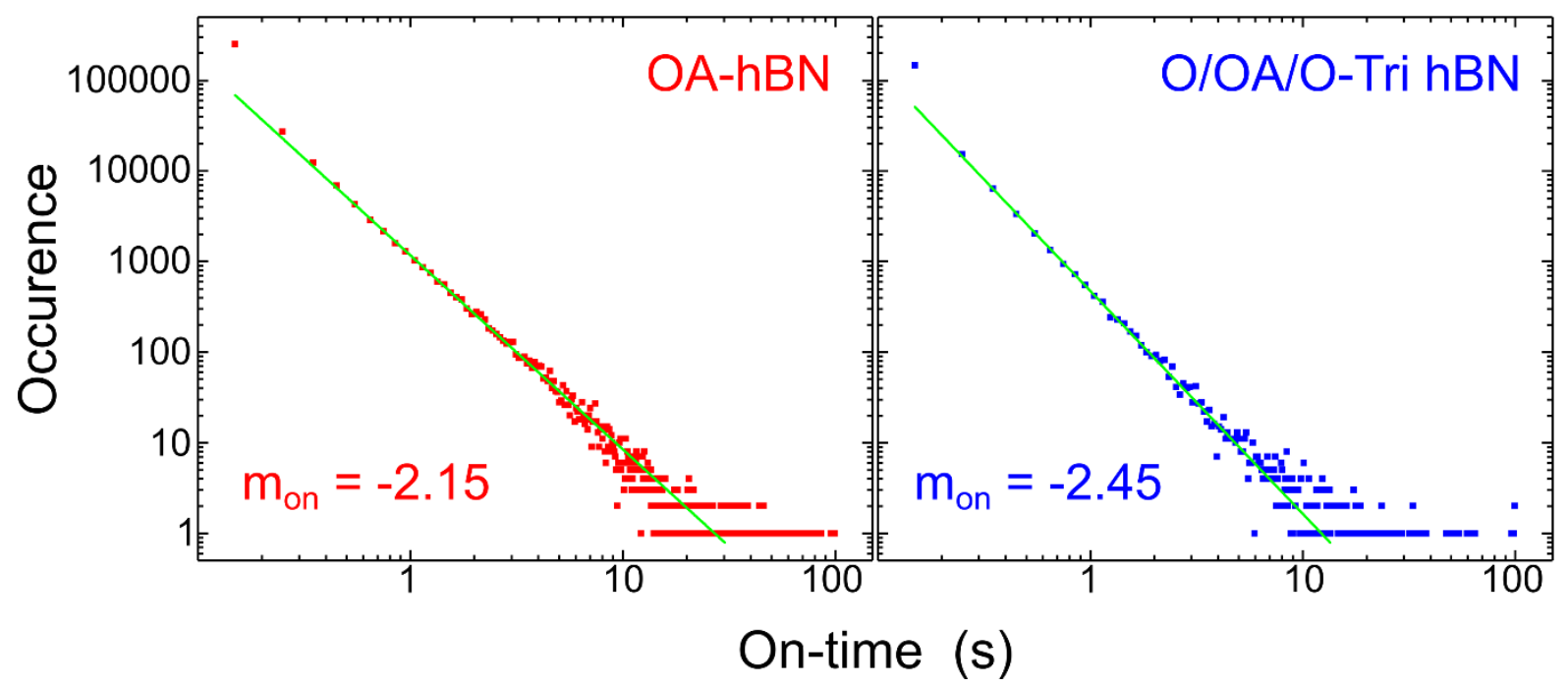

Figure S11: Blinking Statistics: Log-log plots of the occurrence of on-times for both the OA-hBN (red) and $O / O A / O-T r i h B N$ (blue) fit using a power law function $\mathrm{P}(\mathrm{t}) \propto(\mathrm{t})^{\mathrm{m}}$ (green line). With the power law coefficient $\left(m_{o n}\right)$ shown for each sample.

On-times measure an alternate stability profile to that of bleaching, as mentioned in the main text. Blinking is a phenomenon ubiquitous to SPE, where bleaching is a permanent turning off the emission site, blinking is fluorescent intermittency caused by the fluctuating charge state of an emission site and its surrounding environment. To generate on-times the 1D array of each emitter's lifetime (see Methods) was used to determine how many consecutive frames it was "on"/ "bright" for. The number of consecutive frames (frame length $100 \mathrm{~ms}$ ) is the on-time, and these on-times were binned into 100 $\mathrm{ms}$ and plotted on a log-log scale of occurrence to on time, shown in Fig. S9. A power law function was fitted: $P(t) \propto(t)^{m}$ where $t$ is the on-time, and $m$ is the power law coefficient used to measure the stability of the sample. The values found are comparable to previously reported work on CVD grown hBN ML., The trilayer $h-B N$ stack's $m_{\text {on }}$ does not increase, meaning that they do not improve blinking stability. Further work is required in neutralising the charge environment, either through substrate or encapsulation optimisation.

\section{Defect Assignment}

The table below summarises the suggested defects that match current experimental data on single photon quantum emitters in hBN in the visible range, particularly focussing on quantum emitters as found in the CVD grown sample of this publication. Our results are consistent with other experimental data ${ }^{5-9}$ on the most dominant emitter type that has been found in CVD grown $\mathrm{hBN}$ with a ZPL between 2.1-2.3 eV together with a phonon side band about $160 \mathrm{meV}$ lower in energy. If the references contain both experimental and theoretical information on the ZPL of the emitters, both values are given. For purely theoretical papers based on the experimental results of others only the theoretical value is given. DFT calculations have significant error margins especially for calculating the nature of the ground state and the absolute energy difference between ground and first excited state. In particular open-shell compared to closed-shell singlet states are predicted to be too low in energy by the order of $1 \mathrm{eV}^{10}$. The results of the ZPL line energy for different calculations of the same defect display a range of energies from which the overall accuracy for this absolute energy difference between ground and first excited sate can be inferred. Further defect structure confirmation can be concluded when an emission sites optically detected magnetic resonance is observed, as has been the case for the $\mathrm{V}_{\mathrm{B}}{ }^{-{ }^{-11-}}$ ${ }^{13}$ Unfortunately, although significant advances have been made on more detailed understandings of 
the range of defects studied in this report, ${ }^{14-16}$ no conclusive confirmation of its exact nature have been made.

\begin{tabular}{|c|c|c|c|}
\hline $\begin{array}{l}\text { ZPL } \\
\text { Experiment/eV(nm) }\end{array}$ & ZPL Theory/eV(nm) & Emitter Type & Reference \\
\hline - & $2.12(585)$ & $\mathrm{V}_{\mathrm{N}} \mathrm{N}_{\mathrm{B}}$ & 10 \\
\hline - & & $\begin{array}{l}\mathrm{V}_{\mathrm{N}} \mathrm{N}_{\mathrm{B}} \text { (ruled out } \\
\text { because of phonon } \\
\text { sideband structure) }\end{array}$ & 17 \\
\hline- & $2.39(518)$ & $\mathrm{V}_{\mathrm{N}} \mathrm{N}_{\mathrm{B}}$ & 18 \\
\hline- & $2.05(605), 2.98(416)$ & $\mathrm{V}_{\mathrm{N}} \mathrm{N}_{\mathrm{B}}$ & 19 \\
\hline $\begin{array}{l}\text { Emitters between } \\
540-820 \mathrm{~nm}) \text { with } \\
\text { peak in histogram } \\
\text { at } 1.88(659) \text { and } \\
2.16(574)\end{array}$ & $2.10(590)$ & $\mathrm{V}_{\mathrm{N}} \mathrm{N}_{\mathrm{B}}$ & 20 \\
\hline - & $2.408(514)$ & $\mathrm{V}_{\mathrm{N}} \mathrm{N}_{\mathrm{B}}$ & 21 \\
\hline \multirow[t]{3}{*}{$\begin{array}{l}2.18(570)- \\
1.69(730)\end{array}$} & $1.93(642), 2.01(616)$ & $\mathrm{V}_{\mathrm{N}} \mathrm{N}_{\mathrm{B}}$ & 22 \\
\hline & $2.08(596)$ & $\mathrm{V}_{\mathrm{N}} \mathrm{C}_{\mathrm{B}}$ & 10 \\
\hline & $1.39(891)$ & $\mathrm{V}_{\mathrm{N}} \mathrm{C}_{\mathrm{B}}$ & 17 \\
\hline - & 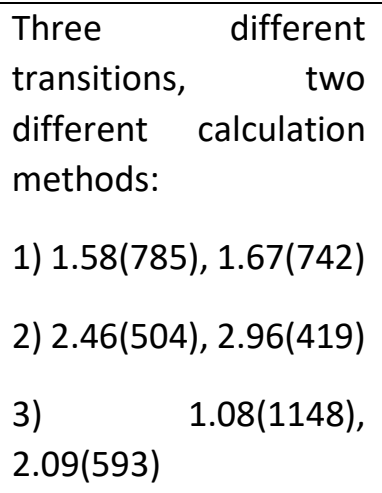 & $\mathrm{V}_{\mathrm{N}} \mathrm{C}_{\mathrm{B}}$ & 18 \\
\hline - & $1.75(708)$ & $\mathrm{V}_{\mathrm{N}} \mathrm{C}_{\mathrm{B}}$ & 23 \\
\hline $\begin{array}{l}\text { Emitters between } \\
540-820 \mathrm{~nm}) \text { with } \\
\text { peak in histogram } \\
\text { at } 1.88(659) \text { and } \\
2.16(574)\end{array}$ & $\begin{array}{l}\text { Emitter assignment } \\
\text { based on numerical } \\
\text { results and group } \\
\text { theory analysis }\end{array}$ & $\mathrm{V}_{\mathrm{N}} \mathrm{C}_{\mathrm{B}}$ & 20 \\
\hline- & $\begin{array}{ll}2.25(550) & \text { triplet } \\
\text { ground state } & \end{array}$ & $\mathrm{V}_{\mathrm{N}} \mathrm{C}_{\mathrm{B}}$ & 24 \\
\hline
\end{tabular}




\begin{tabular}{|c|c|c|c|}
\hline $2.2(563)$ & $\begin{array}{l}\text { DFT to match spectral } \\
\text { shape }\end{array}$ & $\mathrm{V}_{\mathrm{N}} \mathrm{C}_{\mathrm{B}}$ & 25 \\
\hline - & $1.92(646)$ & $\begin{array}{l}\mathrm{V}_{\mathrm{B}}^{-} \\
\text {Note: This defect } \\
\text { has recently been } \\
\text { assigned to broad } \\
\text { photoluminescence } \\
\text { around } 850 \mathrm{~nm} \text { and } \\
\text { a triplet ground } \\
\text { state. }{ }^{11-13}\end{array}$ & 19 \\
\hline $2(619)$ & $\begin{array}{l}\text { DFT to match spectral } \\
\text { shape }\end{array}$ & $\mathrm{V}_{\mathrm{B}}^{-}$ & 25 \\
\hline $2.12(585)$ & $\begin{array}{l}\text { Calculations to match } \\
\text { lineshape determine } \\
\text { emitter type }\end{array}$ & $\mathrm{V}_{\mathrm{B}} \mathrm{C}_{\mathrm{N}}^{-}$ & 16 \\
\hline - & $1.93(642)$ & $\mathrm{V}_{\mathrm{B}} \mathrm{C}_{\mathrm{N}}$ & 17 \\
\hline- & $2.06(601)$ & $\begin{array}{l}\text { Boron dangling } \\
\text { bonds B DB- }\end{array}$ & $\overline{26}$ \\
\hline $\begin{array}{l}6 \text { experimental ZPL } \\
\text { peaks: } \\
\text { P1:2.25(551) } \\
\text { P2: 2.15(576) } \\
\text { P3: 1.98(626) } \\
\text { P4: 1.90(653) } \\
\text { P5: 1.81(685) } \\
\text { P6: 1.69(734) } \\
\text { Highest peaks in } \\
\text { histogram for } \\
\text { different samples: } \\
\text { Liquid exfoliated: } \\
\text { P2 } \\
\text { multilayer cvd: P2 } \\
\text { monolayer cvd: P3 }\end{array}$ & $\begin{array}{l}2.298(539)-2.188(566) \\
(n=8,9,14,15,16) \\
2.404(515)(n=10)\end{array}$ & $\begin{array}{l}\text { Triangular defects } \\
\text { with multiple } \\
\text { number }(n) \text { of } B \text { and } \\
N \text { atoms missing }\end{array}$ & 27 \\
\hline
\end{tabular}




\begin{tabular}{|c|c|c|c|}
\hline - & $2.03(610)$ & $\mathrm{V}_{\mathrm{B}} \mathrm{C}_{N} \mathrm{Si}_{\mathrm{N}}$ & 10 \\
\hline - & 1.90 (652) & $\mathrm{V}_{\mathrm{N}} \mathrm{O}_{2 \mathrm{~B}}$ & 10 \\
\hline - & $\begin{array}{l}1.63(760) \\
\text { Calculation gives a ZPL- } \\
\text { PSB separation of } \\
160 \mathrm{meV}\end{array}$ & {$\left[C_{N}\right]_{2} C_{B}$} & 28 \\
\hline - & $\begin{array}{l}1.65(751) \\
\text { Calculation gives a ZPL- } \\
\text { PSB separation of } \\
160 \mathrm{meV}\end{array}$ & $C_{N}\left[C_{B}\right]_{2}$ & 28 \\
\hline peak at (2.29)540 & & $\begin{array}{lr}\text { production } & \text { in } \\
\text { carbon } & \text { rich } \\
\text { atmosphere } & \end{array}$ & 29 \\
\hline
\end{tabular}

Table S2: Previously reported assignments of the structural origin of defects responsible for SPE in $h B N$ and their corresponding wavelengths.

\section{References}

(1) Prosanov, I. Yu.; Matvienko, A. A. Study of PVA Thermal Destruction by Means of IR and Raman Spectroscopy. Physics of the Solid State 2010, 52 (10), 2203-2206. https://doi.org/10.1134/S1063783410100318.

(2) Dollish, F. R.; Fateley, W. G.; Bentley, F. F. Characteristic Raman Frequencies of Organic Compounds; Wiley: New York, 1974.

(3) Comtet, J.; Glushkov, E.; Navikas, V.; Feng, J.; Babenko, V.; Hofmann, S.; Watanabe, K.; Taniguchi, T.; Radenovic, A. Wide-Field Spectral Super-Resolution Mapping of Optically Active Defects in Hexagonal Boron Nitride. Nano Lett. 2019, 19 (4), 2516-2523. https://doi.org/10.1021/acs.nanolett.9b00178.

(4) Stern, H. L.; Wang, R.; Fan, Y.; Mizuta, R.; Stewart, J. C.; Needham, L.-M.; Roberts, T. D.; Wai, R.; Ginsberg, N. S.; Klenerman, D.; Hofmann, S.; Lee, S. F. Spectrally Resolved Photodynamics of Individual Emitters in Large-Area Monolayers of Hexagonal Boron Nitride. ACS Nano 2019, 13 (4), 4538-4547. https://doi.org/10.1021/acsnano.9b00274.

(5) Mendelson, N.; Xu, Z.-Q.; Tran, T. T.; Kianinia, M.; Scott, J.; Bradac, C.; Aharonovich, I.; Toth, $M$. Engineering and Tuning of Quantum Emitters in Few-Layer Hexagonal Boron Nitride. ACS Nano 2019, 13 (3), 3132-3140. https://doi.org/10.1021/acsnano.8b08511.

(6) Chejanovsky, N.; Rezai, M.; Paolucci, F.; Kim, Y.; Rendler, T.; Rouabeh, W.; Fávaro de Oliveira, F.; Herlinger, P.; Denisenko, A.; Yang, S.; Gerhardt, I.; Finkler, A.; Smet, J. H.; Wrachtrup, J. Structural Attributes and Photodynamics of Visible Spectrum Quantum Emitters in Hexagonal Boron Nitride. Nano Lett. 2016, 16 (11), 7037-7045. https://doi.org/10.1021/acs.nanolett.6b03268.

(7) Li, C.; Mendelson, N.; Ritika, R.; Chen, Y.; Xu, Z.-Q.; Toth, M.; Aharonovich, I. Scalable and Deterministic Fabrication of Quantum Emitter Arrays from Hexagonal Boron Nitride. Nano Lett. 2021, 21 (8), 3626-3632. https://doi.org/10.1021/acs.nanolett.1c00685.

(8) Nikolay, N.; Mendelson, N.; Özelci, E.; Sontheimer, B.; Böhm, F.; Kewes, G.; Toth, M.; Aharonovich, I.; Benson, O. Direct Measurement of Quantum Efficiency of Single-Photon 
Emitters in Hexagonal Boron Nitride. Optica 2019, 6 (8), 1084.

https://doi.org/10.1364/OPTICA.6.001084.

(9) Glushkov, E.; Mendelson, N.; Chernev, A.; Ritika, R.; Lihter, M.; Zamani, R. R.; Comtet, J.; Navikas, V.; Aharonovich, I.; Radenovic, A. Direct Growth of Hexagonal Boron Nitride on Photonic Chips for High-Throughput Characterization. 2021 arXiv:2103.15415 [cond-mat, physics:physics]. http://arxiv.org/abs/2103.15415 (accessed July 6, 2021)

(10) Sajid, A.; Reimers, J. R.; Ford, M. J. Defect States in Hexagonal Boron Nitride: Assignments of Observed Properties and Prediction of Properties Relevant to Quantum Computation. Phys. Rev. B 2018, 97 (6), 064101. https://doi.org/10.1103/PhysRevB.97.064101.

(11) Gottscholl, A.; Kianinia, M.; Soltamov, V.; Orlinskii, S.; Mamin, G.; Bradac, C.; Kasper, C.; Krambrock, K.; Sperlich, A.; Toth, M.; Aharonovich, I.; Dyakonov, V. Initialization and Read-Out of Intrinsic Spin Defects in a van der Waals Crystal at Room Temperature. Nature Materials 2020, 19 (5), 540-545. https://doi.org/10.1038/s41563-020-0619-6.

(12) Gottscholl, A.; Diez, M.; Soltamov, V.; Kasper, C.; Sperlich, A.; Kianinia, M.; Bradac, C.; Aharonovich, I.; Dyakonov, V. Sub-Nanoscale Temperature, Magnetic Field and Pressure Sensing with Spin Centers in 2D Hexagonal Boron Nitride. 2021 arXiv:2102.10890 [cond-mat, physics:quant-ph]. http://arxiv.org/abs/2102.10890 (accessed July 6, 2021)

(13) Toledo, J. R.; de Jesus, D. B.; Kianinia, M.; Leal, A. S.; Fantini, C.; Cury, L. A.; Sáfar, G. A. M.; Aharonovich, I.; Krambrock, K. Electron Paramagnetic Resonance Signature of Point Defects in Neutron-Irradiated Hexagonal Boron Nitride. Phys. Rev. B 2018, 98 (15), 155203. https://doi.org/10.1103/PhysRevB.98.155203.

(14) Chejanovsky, N.; Mukherjee, A.; Geng, J.; Chen, Y.-C.; Kim, Y.; Denisenko, A.; Finkler, A.; Taniguchi, T.; Watanabe, K.; Dasari, D. B. R.; Auburger, P.; Gali, A.; Smet, J. H.; Wrachtrup, J. Single-Spin Resonance in a van der Waals Embedded Paramagnetic Defect. Nature Materials 2021. https://doi.org/10.1038/s41563-021-00979-4.

(15) Stern, H. L.; Jarman, J.; Gu, Q.; Barker, S. E.; Mendelson, N.; Chugh, D.; Schott, S.; Tan, H. H.; Sirringhaus, H.; Aharonovich, I.; Atatüre, M. Room-Temperature Optically Detected Magnetic Resonance of Single Defects in Hexagonal Boron Nitride. 2021 arXiv:2103.16494 [cond-mat, physics:quant-ph]. http://arxiv.org/abs/2103.16494 (accessed April 23, 2021)

(16) Mendelson, N.; Chugh, D.; Reimers, J. R.; Cheng, T. S.; Gottscholl, A.; Long, H.; Mellor, C. J.; Zettl, A.; Dyakonov, V.; Beton, P. H.; Novikov, S. V.; Jagadish, C.; Tan, H. H.; Ford, M. J.; Toth, M.; Bradac, C.; Aharonovich, I. Identifying Carbon as the Source of Visible Single-Photon Emission from Hexagonal Boron Nitride. Nature Materials 2021, 20 (3), 321-328. https://doi.org/10.1038/s41563-020-00850-y.

(17) Tawfik, S. A.; Ali, S.; Fronzi, M.; Kianinia, M.; Tran, T. T.; Stampfl, C.; Aharonovich, I.; Toth, M.; Ford, M. J. First-Principles Investigation of Quantum Emission from HBN Defects. Nanoscale 2017, 9 (36), 13575-13582. https://doi.org/10.1039/C7NR04270A.

(18) Sajid, A.; Ford, M. J.; Reimers, J. R. Single-Photon Emitters in Hexagonal Boron Nitride: A Review of Progress. Rep. Prog. Phys. 2020, 83 (4), 044501. https://doi.org/10.1088/13616633/ab6310.

(19) Abdi, M.; Chou, J.-P.; Gali, A.; Plenio, M. B. Color Centers in Hexagonal Boron Nitride Monolayers: A Group Theory and ab Initio Analysis. ACS Photonics 2018, 5 (5), 1967-1976. https://doi.org/10.1021/acsphotonics.7b01442.

(20) Yim, D.; Yu, M.; Noh, G.; Lee, J.; Seo, H. Polarization and Localization of Single-Photon Emitters in Hexagonal Boron Nitride Wrinkles. ACS Appl. Mater. Interfaces 2020, 12 (32), 36362-36369. https://doi.org/10.1021/acsami.0c09740.

(21) Smart, T. J.; Li, K.; Xu, J.; Ping, Y. Intersystem Crossing and Exciton-Defect Coupling of Spin Defects in Hexagonal Boron Nitride. npj Computational Materials 2021, 7 (1), 1-8. https://doi.org/10.1038/s41524-021-00525-5. 
(22) Xue, Y.; Wang, H.; Tan, Q.; Zhang, J.; Yu, T.; Ding, K.; Jiang, D.; Dou, X.; Shi, J.; Sun, B. Anomalous Pressure Characteristics of Defects in Hexagonal Boron Nitride Flakes. ACS Nano 2018, 12 (7), 7127-7133. https://doi.org/10.1021/acsnano.8b02970.

(23) Sajid, A.; Thygesen, K. S. $V_{N} C_{B}$ Defect as Source of Single Photon Emission from Hexagonal Boron Nitride. 2D Mater. 2020, 7 (3), 031007. https://doi.org/10.1088/2053-1583/ab8f61.

(24) Cheng, G. D.; Zhang, Y. G.; Yan, L.; Huang, H. F.; Huang, Q.; Song, Y. X.; Chen, Y.; Tang, Z. A Paramagnetic Neutral $C_{B} V_{N}$ Center in Hexagonal Boron Nitride Monolayer for Spin Qubit Application. Computational Materials Science 2017, 129, 247-251. https://doi.org/10.1016/j.commatsci.2016.12.032.

(25) Fischer, M.; Caridad, J. M.; Sajid, A.; Ghaderzadeh, S.; Ghorbani-Asl, M.; Gammelgaard, L.; Bøggild, P.; Thygesen, K. S.; Krasheninnikov, A. V.; Xiao, S.; Wubs, M.; Stenger, N. Controlled Generation of Luminescent Centers in Hexagonal Boron Nitride by Irradiation Engineering. Science Advances 2021, 7 (8), eabe7138. https://doi.org/10.1126/sciadv.abe7138.

(26) Turiansky, M. E.; Van de Walle, C. G. Boron Dangling Bonds in a Monolayer of Hexagonal Boron Nitride. Journal of Applied Physics 2021, 129 (6), 064301. https://doi.org/10.1063/5.0040780.

(27) Kozawa, D.; Rajan, A. G.; Li, S. X.; Ichihara, T.; Koman, V. B.; Zeng, Y.; Kuehne, M.; Iyemperumal, S. K.; Silmore, K. S.; Parviz, D.; Liu, P.; Liu, A. T.; Faucher, S.; Yuan, Z.; Xu, W.; Warner, J. H.; Blankschtein, D.; Strano, M. S. Observation and Spectral Assignment of a Family of Hexagonal Boron Nitride Lattice Defects. 2019 arXiv:1909.11738 [cond-mat, physics:quantph]. http://arxiv.org/abs/1909.11738 (accessed Oct. 1, 2019)

(28) Jara, C.; Rauch, T.; Botti, S.; Marques, M. A. L.; Norambuena, A.; Coto, R.; Castellanos-Águila, J. E.; Maze, J. R.; Munoz, F. First-Principles Identification of Single Photon Emitters Based on Carbon Clusters in Hexagonal Boron Nitride. J. Phys. Chem. A 2021, 125 (6), 1325-1335. https://doi.org/10.1021/acs.jpca.0c07339.

(29) Lyu, C.; Zhu, Y.; Gu, P.; Qiao, J.; Watanabe, K.; Taniguchi, T.; Ye, Y. Single-Photon Emission from Two-Dimensional Hexagonal Boron Nitride Annealed in a Carbon-Rich Environment. Appl. Phys. Lett. 2020, 117 (24), 244002. https://doi.org/10.1063/5.0025792. 